\title{
The Contribution of the Left Inferior Frontal Gyrus in Affective Processing of Social Groups
}

Tiziano Suran

Area of Neuroscience, SISSA, Via Bonomea 265, 34136 Trieste, Italy

ORCiD:_https://orcid.org/0000-0002-5471-8929

Raffaella I. Rumiati

ANVUR, Via Ippolito Nievo 35, 00153 Rome, Italy

Area of Neuroscience, SISSA, Via Bonomea 265, 34136 Trieste, Italy

ORCiD: https://orcid.org/0000-0002-3836-8295

Luca Piretti

Area of Neuroscience, SISSA, Via Bonomea 265, 34136 Trieste, Italy

Department of Psychology and Cognitive Sciences, University of Trento, Corso Bettini 84, 38068, Rovereto, Italy

Fondazione ONLUS Marica De Vincenzi, Rovereto, Italy

ORCiD: https://orcid.org/0000-0002-9304-9780

Corresponding author:

Tiziano Suran

Area of Neuroscience, SISSA

Via Bonomea 265

34136 Trieste, Italy

Email: tiziano.suran@gmail.com

Declarations of interest: none to declare. 


\begin{abstract}
We investigated the contribution of the pars opercularis of the left inferior frontal gyrus (LIFGop) in representing knowledge about social groups. We asked healthy individuals to categorize words preceded by semantically congruent or incongruent primes while stimulating the LIFGop. Previous studies showing an involvement of the LIFGop both in processing social stimuli and negative valence words led us to predict that its stimulation would affect responses to negative social category words. Compared to the Vertex as control site, the stimulation of the LIFGop increased the speed of categorization of negative social groups, and disrupted the semantic priming effect for negative words overall. Within the framework of recent theories of semantic memory, we argue that the present results provide initial evidence of the representation of social groups being characterized by affective properties, whose processing is supported by the LIFGop.
\end{abstract}

Keywords: Social Groups; Valence; Transcranial Magnetic Stimulation; Pars opercularis; Category Specificity; Priming. 


\section{INTRODUCTION}

The study of neurological patients' cognitive and affective abilities allows highlighting the underlying neural systems. Thus, brain damage has been shown to selectively and severely impair the ability of individuals to name and recall detailed information about otherwise familiar people, leaving their semantic knowledge about objects or animals intact (e.g., Miceli et al., 2000; Thompson et al., 2004); or it can produce the reverse pattern, that is, impaired recognition of objects and animals with intact knowledge about people (Haslam \& Sabah, 2013; Kay \& Hanley, 2002; Lyons, Kay, Hanley, \& Haslam, 2006; Thompson et al., 2004). These dissociations argue in favor of distinct brain systems for processing conspecifics and other non-social stimuli. However, the theories formulated to explain the category-specific deficits initially reported only among non-social categories have been a little silent on how concepts about conspecifics might be represented (for reviews, see Capitani, Laiacona, Mahon, \& Caramazza, 2003; Caramazza, Anzellotti, Strnad, \& Lingnau, 2014; Thompson-Schill, 2003).

Extant neuropsychological research has shown that patients' failure to name/recognize famous people such as Ronald Reagan (Miceli et al., 2000; Thompson et al., 2004) is selectively limited to the individual identities, while their ability to recognize non-social entities remains intact. Nevertheless, the level of the information required for the successful recognition of individual conspecifics might be intrinsically different from the one required for recognizing non-social entities. Recently, Rumiati, Piretti and colleagues reported that some patients were selectively impaired at recognizing conspecifics when presented as social groups (e.g., doctors; Piretti et al., 2015; Rumiati, Carnaghi, Improta, Diez, \& Silveri, 2014). This deficit is argued to originate at the semantic level as it has been observed when either sorting names of social groups (Rumiati et al., 2014) or naming images depicting them (Piretti et al., 2015). In the latter study, using voxel-based lesion-symptom mapping (VLSM), Piretti et al. (2015) documented tumor patients with lesions in regions associated 
also with affective processes such as insula, amygdala, basal ganglia, and inferior frontal gyrus, who were impaired at naming photographs of social groups. This association between damage to affective processing areas and recognition deficits involving social groups led us to hypothesize that successful recognition of such social categories may depend on an intact ability to process affective information. As the recognition of living and non-living things primarily weights on sensory and functional properties respectively, we hypothesized that recognition of social groups might require a privileged access to affective properties. Indeed, compared with patients without the deficit and healthy controls, patients with dementia and semantic deficits for social groups expressed less extreme affective evaluations for both positive and negative social groups, an effect that is known as emotional blunting (Carnaghi, Silveri, \& Rumiati, 2015).

From a theoretical perspective, Barsalou and colleagues proposed that, in addition to the other sensory modalities, a model of conceptual knowledge should also include an emotion modality that is relevant for processing the affective properties of conceptual representations (Simmons and Barsalou, 2003; Barsalou, 2008). In their view, the internal affective states represent a component of knowledge that is as important as the external perceptual experience processed by other modalities (Barsalou, 2008). Thus, the patients' deficit at recognizing social groups (Rumiati et al., 2014; Piretti et al., 2015) can be interpreted as being due to degraded affective properties that play a prominent role in their representation. Martin (2016) too suggested that the affective properties are stored in emotional systems, with affective information being a salient component of social concepts. An emotion modality is also present in the hub-and-spokes model proposed by Lambon Ralph and collaborators (Lambon Ralph, Jefferies, Patterson, \& Rogers, 2016; Noonan, Jefferies, Visser, \& Lambon Ralph, 2013; Patterson, Nestor, \& Rogers, 2007; Rogers et al., 2004) whereby the conceptual representations result from integrating several, modality-specific areas or spokes - that process information related to sound, praxis, function, vision, valence, and verbal description - which in turn feed a central modality-independent module (the ' $h u b$ '). Interestingly, the model predicts that 
impairments involving the representation of conspecifics may derive from damage to cortical areas that process valence (Lambon Ralph et al., 2016).

As to the neural correlates, the pars opercularis of the left inferior frontal gyrus (LIFGop) singled out by Piretti et al (2015) is one of the candidates in which the affective processing of social groups may take place. This area has also been found across several neuroimaging studies. First, the LIFGop is specifically involved in the semantic representation of familiar people when individual identities are categorized based on their profession at the level of social group (Chedid et al., 2016). Additionally, the LIFGop is one of the joint regions being active when healthy subjects read sentences describing behaviors of both individuals and social groups (Van der Cruyssen, Heleven, Ma, Vandekerckhove, \& Van Overwalle, 2014).

As to the involvement in the processing of affective information related to conspecifics, the LIFGop has been associated with both emotional empathic responses (Shamay-Tsoory, AharonPeretz, \& Perry, 2009), and in processing emotional facial expressions (Decety \& Chaminade, 2003). Moreover, as the LIFGop is also part of Broca's area, its damage has also been shown to impair linguistic processes linked to affect. Thus damage to this region impairs the retrieval of the names of the emotions conveyed by facial expressions (Adolphs, Damasio, Tranel, Cooper, \& Damasio, 2000), while neuroimaging techniques repeatedly highlighted a link between LIFGop and the affective processing of words with a negative valence. For instance, the cortical activity in this area increases more when participants judge the animacy of words with negative valence than positive valence (Kensinger \& Schacter, 2006; but see also Leclerc \& Kensinger, 2011). Additionally, a greater LIFGop activation to negative than neutral or positive words has been recorded in working memory tasks with emotional word distractors (García-Pacios, Garcés, del Río, \& Maestú, 2017). Last, the area is increasingly activated when subjects read sentences about negative social groups or an individual with a negative trait performing a negative behavior, compared to when positive behaviors 
are performed by positive social groups or individuals (Van der Cruyssen et al., 2014). In sum, the reviewed evidence suggests a key role of the LIFGop in both lexical and affective processing involving conspecifics. Based on the hypothesis that the representation of social groups relies on processing affective information, and that the LIFGop supports to a greater extent the processing of negative words, this area might in turn be expected to support the representation of social groups with a negative valence.

In the present study we applied transcranial magnetic stimulation (TMS) over the LIFGop when healthy participants performed a semantic priming task. In fact, there is a consensus that the contribution of an area to a category-specific representation can be established by applying TMS during a semantic priming task (e.g., Cattaneo, Devlin, Salvini, Vecchi, \& Silvanto, 2010; Fuggetta, Rizzo, Pobric, Lavidor, \& Walsh, 2009). The stimulation, by influencing the activity of an area based on the initial state of activation, should induce the same facilitatory effects in categorizing an unprimed target as if it was preceded by a congruent prime (Cattaneo, Rota, Vecchi, \& Silvanto, 2008). Consistently, we expected a smaller priming effect for social groups than for objects when stimulating the LIFGop, with no significant differences in RTs between congruent and incongruent trials containing social group targets. More specifically, this reduced difference should result from the categorization of social groups in incongruent trials targets becoming faster when stimulating the LIFGop relative to a control site, leaving the responses in congruent trials unaffected. Given the previously established link between the LIFGop and the processing of negative valence stimuli, we additionally expected to record stronger or unique effects for social group words associated with a more negative valence. 


\section{METHOD}

\section{Participants}

Twenty participants (10 females, age range: 20 - 31 years) took part to the study for a monetary compensation. The inclusion criteria consisted in the absence of common contraindications to TMS (Rossi et al., 2009; Rossi, Hallett, Rossini, \& Pascual-Leone, 2011), speaking Italian as first language and self-reported right-handedness. This study protocol was approved and carried out in accordance with the recommendations of the local Ethics Committee (2793/10), and in accordance with the Declaration of Helsinki. All subjects gave written informed consent prior to participating.

\section{Materials}

Stimuli were selected from a dataset of 175 Italian plural related nouns of Objects (e.g., chiavi [keys]) and Social groups (e.g., studenti [students]). Ratings were collected from a sample of 14 participants, and were based on familiarity, imageability, and valence. The pool of selected stimuli consisted of 30 nouns of Social groups and 30 nouns of Objects. The two categories were matched on word length, familiarity, imageability, and valence. Additionally, each category was divided into two sub-samples of equal size based on their valence. Within each category, Positive (e.g., modelle [female models], gioielli [jewels]) and Negative words (e.g., alcolisti [alcoholics], pistole [guns]) were matched based on the previous parameters (both $p \mathrm{~s}>.05$ ), but differed significantly in their valence (both $p \mathrm{~s}<.05$; see Table 1). The words 'PERSONE' (people) and 'OGGETTI' (objects) were used as primes, with the former being treated as semantically congruent to Social groups, and the latter to Objects. Primes and targets were always presented in uppercase and lowercase, respectively, to make it easier for participants to distinguish between the two and reduce the likelihood of erroneously responding to the primes. 


\begin{tabular}{ccccc}
\hline Category & Valence & Imageability & Familiarity & Length \\
\hline $\begin{array}{c}\text { Social } \\
\text { positive }\end{array}$ & $6.23(.46)$ & $7.03(.42)$ & $6.50(1.12)$ & $7.93(1.79)$ \\
$\begin{array}{c}\text { Object } \\
\text { positive }\end{array}$ & $6.06(.48)$ & $7.36(.83)$ & $5.90(1.33)$ & $7.20(1.47)$ \\
$\begin{array}{c}\text { Social } \\
\text { negative }\end{array}$ & $3.98(.80)$ & $7.04(.38)$ & $5.99(.96)$ & $7.13(1.89)$ \\
$\begin{array}{c}\text { Object } \\
\text { negative }\end{array}$ & $3.45(.76)$ & $7.25(.42)$ & $5.69(1.08)$ & $7.00(1.77)$ \\
\hline
\end{tabular}

Table 1. Mean valence, imageability, and familiarity ratings on a scale from 0 to $9(0=$ extremely low; $9=$ extremely high) and average word length for each word category. Standard deviations reported within parentheses.

To assess whether there were significant differences between each target Category/Valence group in terms of their semantic relationship with the primes, we employed the Italian version of the snaut open online software (http://meshugga.ugent.be/snaut-italian-2/; Mandera, Keuleers, \& Brysbaert, 2017) to extract their respective semantic distances, calculated based on word cooccurrences in large text corpora. An ANOVA on the resulting values using Category and Valence as between factors and Congruence as within factor revealed only a significant main effect of Congruence, $F(1,56)=45.73, p<.001, \eta_{p}^{2}=.45$, characterized by a lower semantic distance for Congruent $(M=.79, S D=.10)$, relative to Incongruent primes $(M=.92, S D=.08)$, with all other effects being nonsignificant (all $p s>.35$ ).

\section{Transcranial Magnetic Stimulation}

Three-dimensional MRI data of each subject were co-registered to the volunteer's cranium to provide a navigational template for the positioning of the TMS coil. The positioning was conducted with the Brainsight software (version 2.1.5; Rogue Research, Montreal Canada) connected to a Polaris Vicra 
Optical Tracking System (Polaris, Northern Digital, Ontario, Canada). The system tracked the coil's position with respect to the head using a stereotactic camera, which senses both the coil and the reflectors located on a strap tied to the subject's head. Stimulation sites were identified on each brain reconstruction based on macro-anatomical landmarks (nasion, inion, lateral canthi, and tragi), and the locations of the induced field and stimulation spots were displayed on the MRI data. LIFGop location was based on the findings of Piretti and colleagues (2015) through MNI coordinates $(x=-48, y=1$, $z=15$ ), whereas Vertex location was set manually as the midpoint between nasion and inion and between the tragi.

Paired-pulse TMS was delivered via two $200^{2}$ units connected to a $70 \mathrm{~mm}$ figure-of-eight coil through a BiStim² module (Magstim Company, Whitland, UK). During the experiment, the coil was held by a mechanical arm. For the LIFGop, the coil was oriented with an angle of approximately $45^{\circ}$ from the nasion-inion line with the handle pointing inwards. For the Vertex, the coil was oriented tangentially to the scalp and perpendicular to the sagittal plane. The two pulses were separated by a $100 \mathrm{~ms}$ interval, considered to induce an inhibition of cortical activity (as in, Chen, et al., 1997; Oshio, et al., 2010; but see also Opie, Ridding, \& Semmler, 2015; Shirota, Sommer, \& Paulus, 2016).

\section{Procedure}

Prior to positioning the coil, each participant's resting motor threshold (RMT) was measured. The determination of the RMT was conducted via observed movement motor threshold estimation (OMMT; Pridmore, Fernandes Filho, Nahas, Liberatos, \& George, 1998), defined as the percentage of total machine output (PTMO) of single-pulse stimulation over the left primary motor cortex (M1; 5 $\mathrm{cm}$ lateral from the Vertex) that evoked 5 out of 10 visible contralateral finger/hand motor responses. Stimulation intensity was then set to $90 \%$ of each subject's RMT. 
After positioning the coil over the target area, participants completed a semantic priming paradigm. A PC running E-Prime (version 2.1, Psychological Tools, Inc) controlled the presentation of the stimuli, the recording of responses, and the onset of the TMS pulses. Stimuli were projected on a white background via a 19" LCD monitor with resolution of $1280 * 1024$ pixels and a screen frame rate of $60 \mathrm{~Hz}$. During each trial, a fixation cross was presented for $500 \mathrm{~ms}$, followed by the presentation of a prime word $(50 \mathrm{~ms})$. The prime was replaced by a blank screen $(100 \mathrm{~ms})$ that preceded the target which remained on the screen for $1500 \mathrm{~ms}$, or until participants gave a response via button press (see Fig. 1).

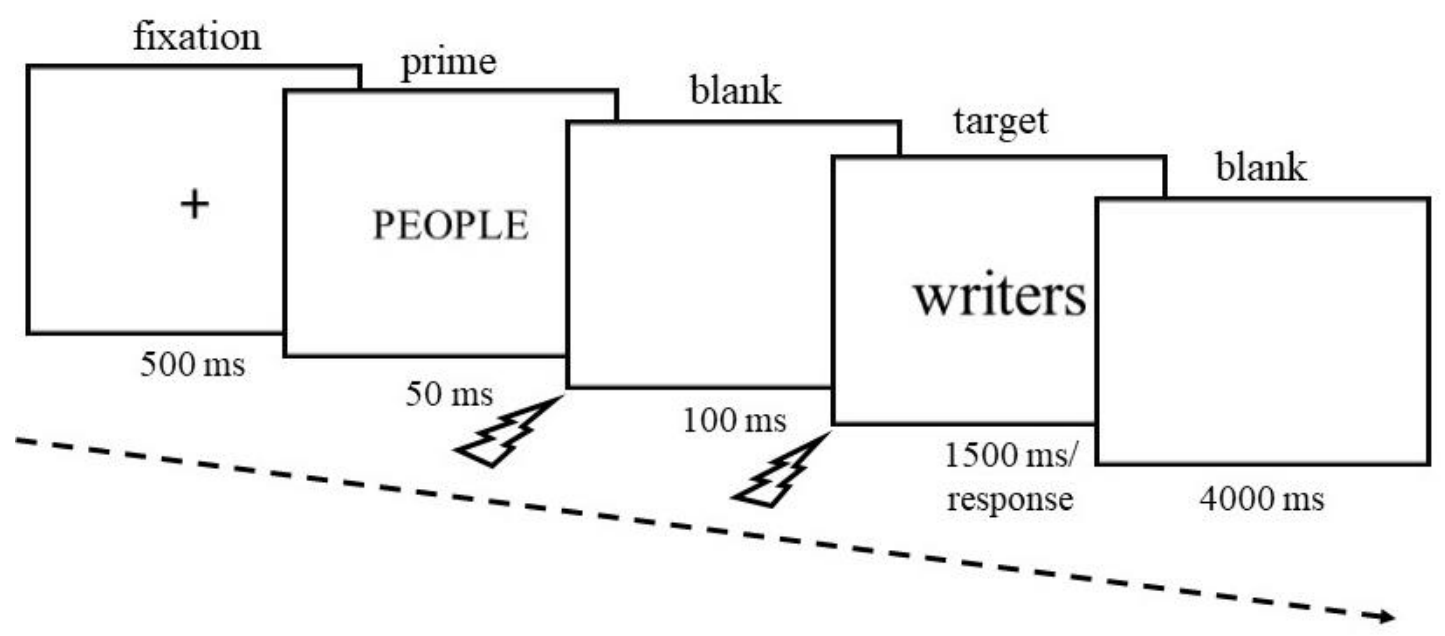

Figure 1. Temporal progression of a trial containing a Positive Social group target preceded by the semantically congruent prime. Bolts indicate TMS pulse delivery, with the first pulse coinciding with the offset of the prime and the second with the onset of the target word, separated by a $100 \mathrm{~ms}$ interval.

Within the task, the first TMS pulse was delivered at the offset of the prime, whereas the second one coincided with the onset of the target word. To avoid potential effects associated to the stimulation in the preceding trial, a $4000 \mathrm{~ms}$ inter-trial interval was used, making a single trial last up to a maximum of $6150 \mathrm{~ms}$. Participants were instructed to just attend the first word (prime) and to categorize the second word (target) based on whether it represented a living or a non-living entity. The response was given via button press, with the category-button mapping counterbalanced across participants. Since the TMS was mainly targeted to a left-lateralized area, participants always 
responded using the index and middle fingers of their left hand. Before starting with the main task, participants were given 5 practice trials with a different sample of stimuli to familiarize with the task and the stimulation. During the main task, all target stimuli were presented twice in a random order, once with the semantically congruent, and once with the semantically incongruent prime. The resulting 120 trials were then split into two blocks of 60 trials each. After the first block, participants could take a short self-paced break, after which they continued by pressing a response button. The same procedure, lasting approximately 7 minutes, was completed by each participant twice, once per stimulation site, with the order of the site being counterbalanced across participants. The experiment thus consisted of a 2 (Site: LIFG vs. Vertex) x 2 (Category: Social groups vs. Objects) x 2 (Valence: Positive vs. Negative) x 2 (prime-target Congruence: Congruent vs. Incongruent) within-subjects design, with response time (RT) as the dependent variable. Given the subjectivity of the valence ratings previously acquired, to check whether the present subjects considered the two semantic categories having an equal valence within each level of the pre-established Valence factor, we asked them to rate the valence of each word at the end of the session.

\section{Data analysis}

We used R (R Core Team, 2012) and lme4 (Bates, Maechler \& Bolker, 2012) to perform a linear mixed effects analysis using RTs as predicted variable and Site, Category, Valence, Congruence and their interactions as fixed effects. The model also controlled for the effects of item length, familiarity and imageability. Random intercepts for each item nested within each subject were used as random effects. Incorrect trials and trials with RTs greater than 2 SD from their average $(629 \mathrm{~ms}, S D=161)$ were filtered prior to analysis. To fix deviations from normality, as from visual inspection of residual plots, RT were transformed via reciprocal log-transformation. Following transformation, no visible deviations from normality we detected. P-values are reported using restricted maximum likelihood 
(REML) estimation and the Satterthwaite approximation to calculate the denominator degrees of freedom.

As a manipulation check, to assess whether the subjects' valence ratings coincided with the pre-determined Valence factor, we ran an ANOVA on the average valence ratings of the target words given at the end of the session, using Category and Valence as factors.

\section{RESULTS}

\section{Main results}

The analysis revealed a significant main effect of Congruence, $F(1,3325)=50.51, p<.001$, with participants being overall slower on Incongruent trials compared to Congruent trials, a three-way interaction between Valence, Category and Site, $F(1,3348)=7.71, p=.006$, and a three-way interaction between Valence, Congruence and Site, $F(1,3328)=3.85, p=.05$. To disentangle the first interaction, we tested how RTs to each Category were influenced by stimulation Site based on whether they were more negative or more positive. To do so, we ran two random effects models, one for each level of Valence, using Category and Site as fixed factors, controlling for imageability, length and familiarity. Random intercepts for Congruence nested within items and subjects were used as random effects.

For Negative stimuli, the results showed a significant Site $\mathrm{x}$ Category interaction, $F(1,1147)$ $=7.19, p=.007$. Bonferroni-corrected pairwise comparisons addressing stimulation effects showed a trend for Social groups, $t(1158)=2.58, p=.06$, Cohen's $d=.24$, with faster responses when stimulating the LIFGop compared to when stimulating the Vertex (see Figure 2). In the case of Positive stimuli, no significant main effects or interactions were observed (all $p s>.10$ ). 

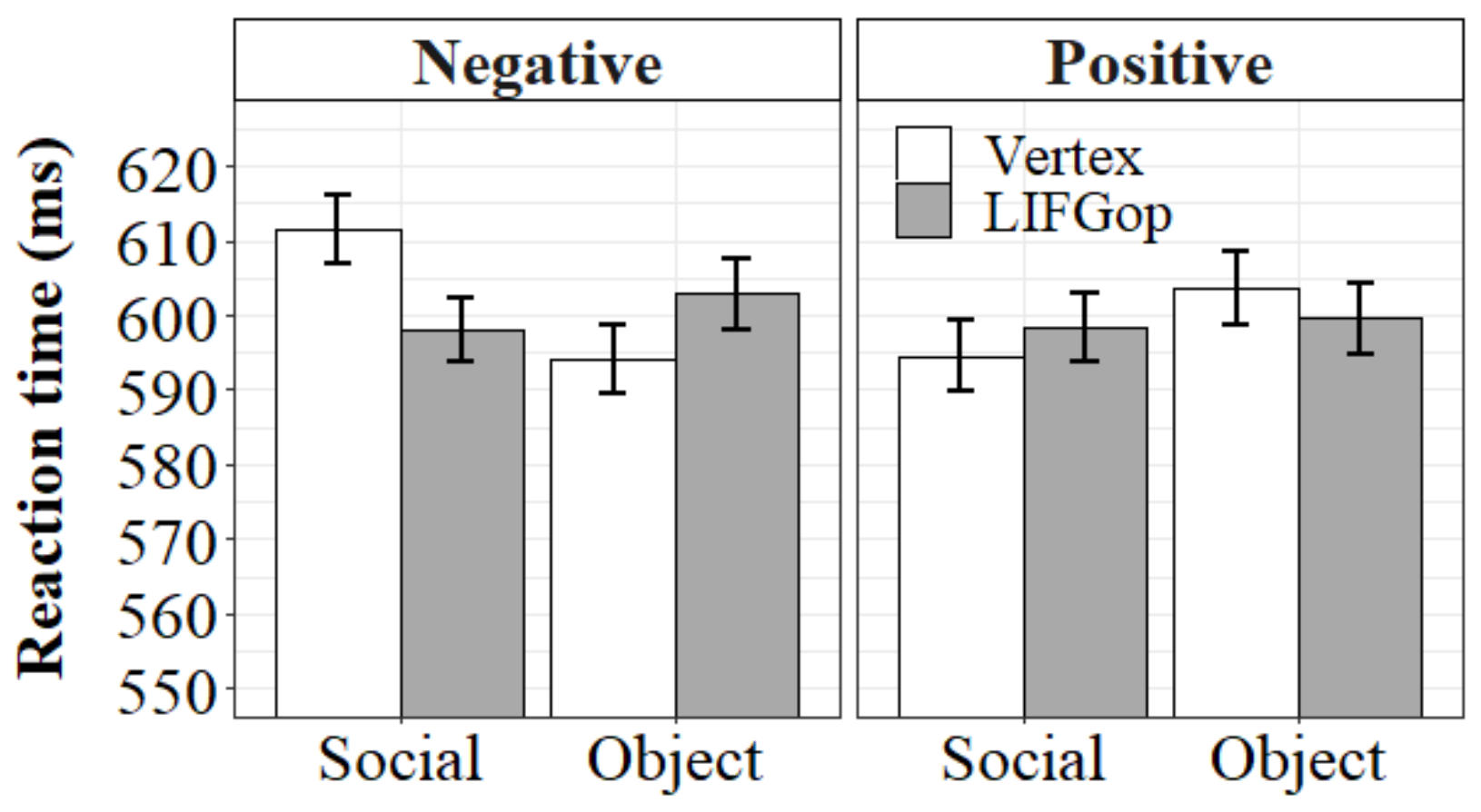

Figure 2. Mean RTs (ms) for Negative (left panel) and Positive targets (right panel) separated by stimulation site and Category. Relative to the Vertex, participants were significantly faster to respond to social groups of more negative valence when the LIFGop was being stimulated. Error bars represent \pm 1 SE.

To disentangle the Valence $\mathrm{x}$ Congruence $\mathrm{x}$ Site interaction, we tested how RTs in each Congruence condition were influenced by the stimulation Site based on whether they belonged to the Negative or Positive valence (see Figure 3). As for the previous interaction, we ran two random effects models, one for each level of Valence, using Congruence and Site as fixed factors, controlling for word imageability, familiarity and valence ratings. Random intercepts for items nested within categories and within subjects were used as random effects.

The results for Negative stimuli showed a main effect of Congruence, $F(1,1679)=12.60, p$ $<.001$, and a marginally significant Site $\mathrm{x}$ Congruence interaction, $F(1,1676)=3.35, p=.07$. Bonferroni-corrected pairwise comparisons showed a significant priming effect during the stimulation of the Vertex, $t(1681)=-3.79, p<.001$, Cohen's $d=-.34$, but not during the stimulation of the LIFGop ( $p>.10$, Cohen's $d=-.11)$. For the Positive valence stimuli, the significant main effect of Congruence, $F(1,1647)=40.41, p<.001$, did not interact with the Site factor $(p>.10)$. 


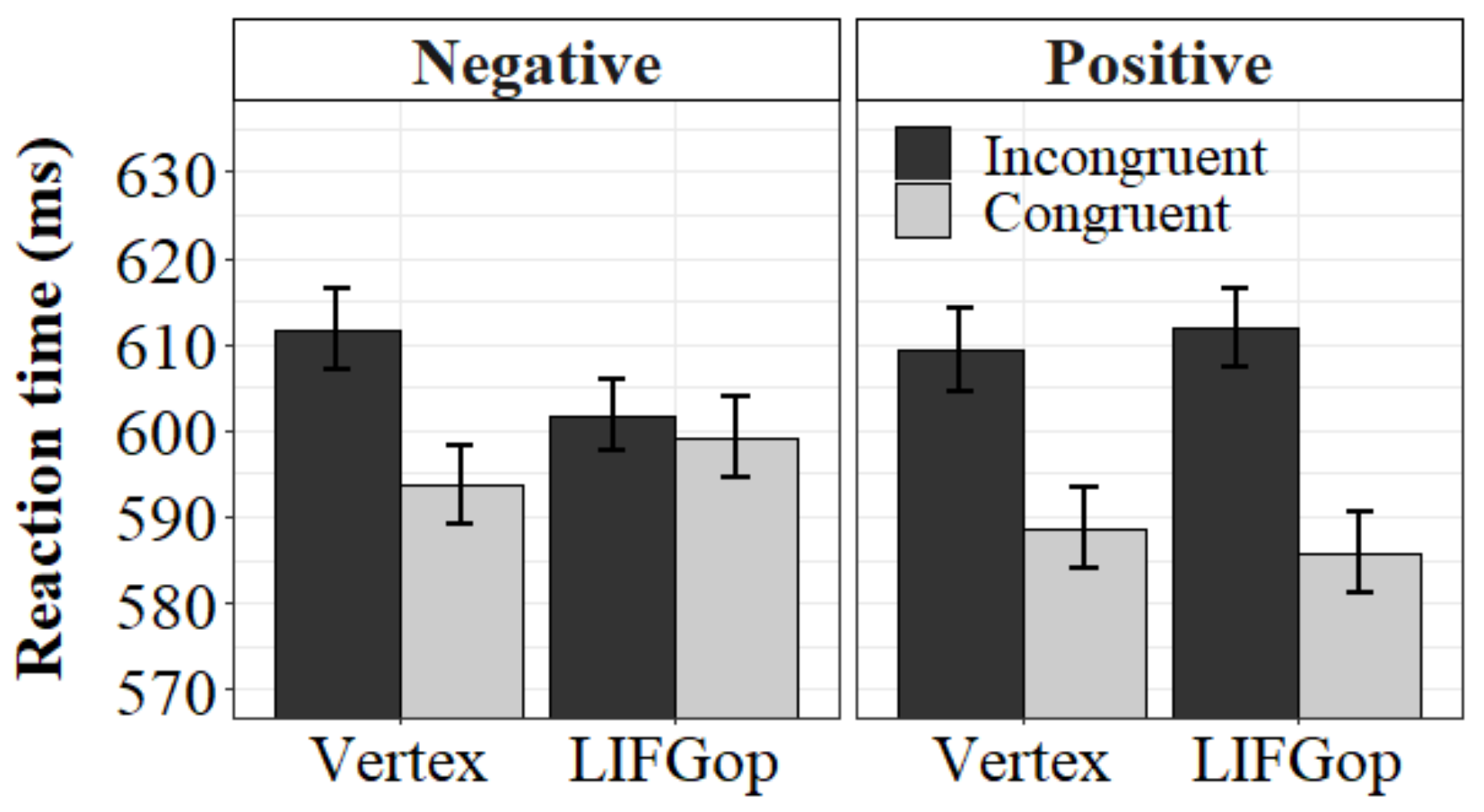

Figure 3. Mean RTs (ms) for Negative (left panel) and Positive targets (right panel) separated by stimulation site and semantic congruence with the preceding prime. Error bars represent $\pm 1 \mathrm{SE}$.

\section{Manipulation check}

The analysis of the valence ratings showed a significant main effect of the pre-established Valence factor, $F(1,19)=65.30, p<.001, \eta^{2}=.77$, with positive valence words having higher ratings $(M=$ $6.29, S D=1.07)$ compared to negative words $(M=4.26, S D=1.20)$. The main effect of Category and the interaction between Category and Valence were not significant (both $p \mathrm{~s}>.10$ ).

\section{Exploratory analysis}

We further explored the data to assess to what extent the faster responses for Negative Social groups during LIFGop stimulation were driven by changes in RTs on Incongruent or Congruent trials relative to the Vertex. To this end, we ran Bonferroni-corrected pairwise comparisons for the Congruence and Site factors separately for Negative Social groups and Negative Objects.

For Negative Social groups, a significant difference between Incongruent and Congruent trials was present when stimulating the Vertex, $t(835)=-2.74, p=.04$, Cohen's $d=-.36$. No difference was 
present when stimulating the LIFGop, $t(835)=-1.08, p>.10$, Cohen's $d=-.14$. The presence of a significant difference when contrasting the Incongruent trials during the Vertex and LIFGop stimulation, $t(837)=2.71, p=.04$, Cohen's $d=.35$, but not the Congruent ones $(p>.10$, Cohen's $d$ $=.14$ ), indicates that the overall faster responses to Negative Social groups, and the same lack of a priming in the LIFGop stimulation condition, was mostly driven by faster responses to unprimed targets (see Figure 4). In the case of Negative Objects, only a marginally significant difference was present between congruent and incongruent trials in the Vertex stimulation condition, $t(842)=-2.57$, $p=.06$, Cohen's $d=-.30$, but not when stimulating the LIFGop ( $\mathrm{p}>.10$, Cohen's $d=-.08$ ).

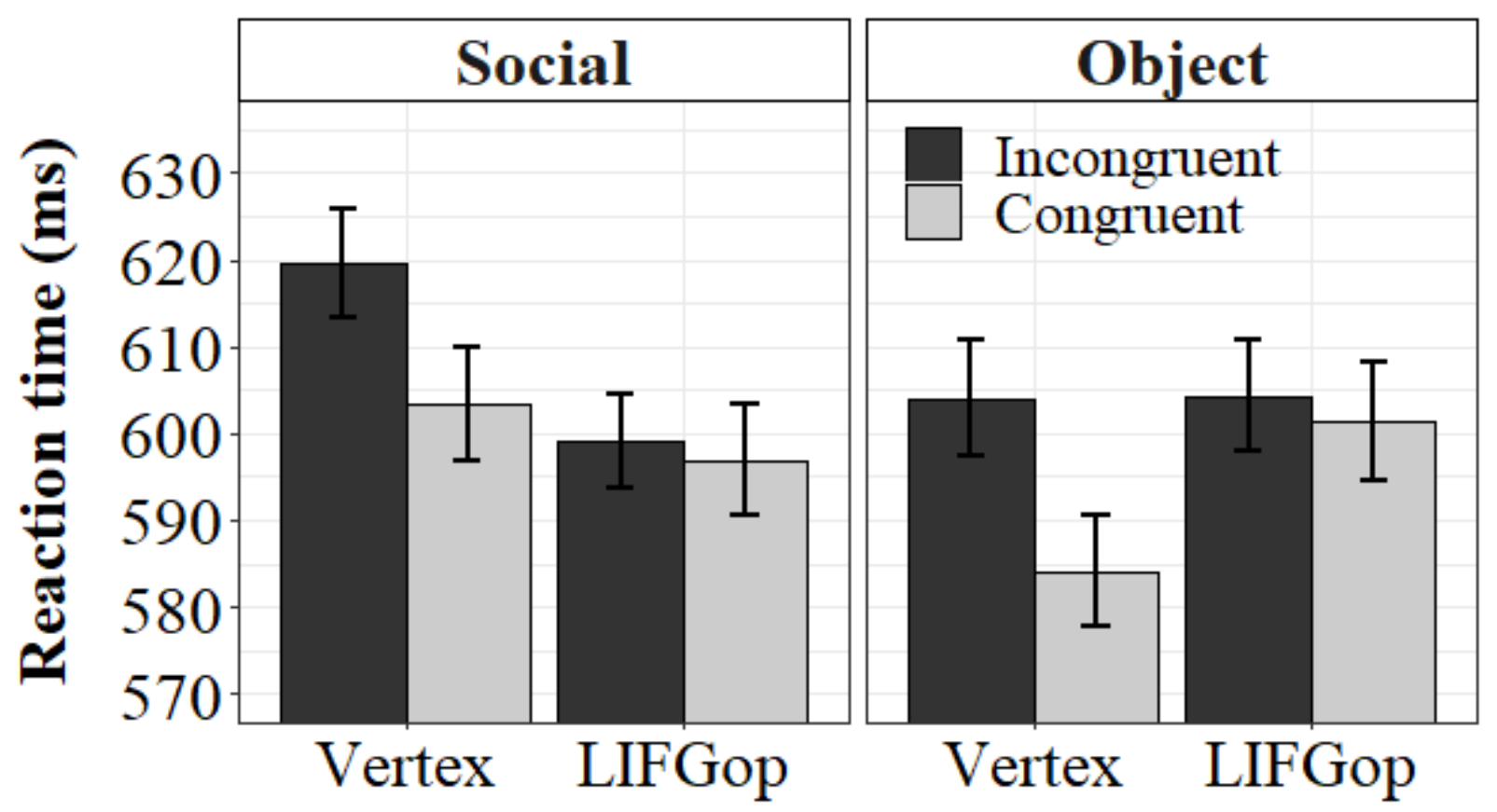

Figure 4. Mean RTs (ms) for Social groups (left panel) and Objects (right panel) of negative valence divided by semantic congruence with the preceding primes and site of stimulation. Error bars represent $\pm 1 \mathrm{SE}$.

\section{DISCUSSION}

With the present study we aimed at assessing the role played by the LIFGop in representing social groups. This prediction was based on empirical facts and extant theories. First, brain-damaged patients were described with a selective deficit in processing social groups (Rumiati et al., 2014), and 
the LIFGop was identified as the underlying common cortical region associated with patients' poorer naming performance on social groups (Piretti et al., 2015). Second, some theoretical propositions (Barsalou, 2008; Lambon Ralph et al., 2016; Martin, 2016) led us to hypothesize that the semantic representation of social groups might be better captured by affective properties, with the affective valence playing a critical role. Moreover, the observed association between LIFGop activity and the processing of negative valence words (García-Pacios et al., 2017; Kensinger \& Schacter, 2006; Leclerc \& Kensinger, 2011) suggested that this area might be specifically involved in representing social groups of negative affective valence.

When TMS was applied over the LIFGop, relative to the Vertex, we found a significant decrease in RTs to negative social categories, with no effect for positive social categories or objects of any valence. Previous TMS studies reported a specific facilitatory effect in unprimed trials (Cattaneo et al., 2010), thus arguing in favor of a TMS-induced increase in cortical excitability based on the initial state of activation (Silvanto, Muggleton, \& Walsh, 2008). The same pattern of results would emerge if the LIFGop were also implied in processing the prime word. In the present case, however, although the effect was mostly led by unprimed trials, faster responses to social groups occurred regardless of the prime congruence. If the LIFGop is implicated in processing negative valence words, the result may be explained by the prime 'people' not producing an activation of that area. On the contrary, the TMS-induced increase in cortical excitability of the LIFGop may have led to an additional facilitatory effect to the one produced by the congruent prime, but affecting only negative social categories.

Additionally, our results have shown a disruption of the priming effect, which, contrary to our expectations, was not unique to social groups, but it was present for negative words in general, including objects. In the latter case, although not significant, the disruption of the priming was numerically mostly led by a decrease in RTs for primed object targets, contrary to what was found 
for social groups. If the LIFGop supports the activation of negative affective representations and these are more strongly linked to the processing of social groups, then an increase in its excitability through stimulation might have increased participants' readiness to categorize a social group when reading a negative word regardless of its category. We hypothesize that such readiness might have produced a lag in the categorization of primed object targets. Although purely speculative, this idea is in line with electrophysiological studies suggesting the presence of effects driven by the affective content of words preceding and influencing later semantic processing (Kissler, Assadollahi, \& Herbert, 2006).

The present findings contain an initial evidence in support of the hypothesis on the relevance of affective information in accessing the representation of social groups, consistently with the proposal that this type of concepts is characterized by a predominance of affective properties (Piretti et al., 2015). This conclusion is consistent with the role of LIFGop in processing negative valence words (García-Pacios et al., 2017; Kensinger \& Schacter, 2006; Leclerc \& Kensinger, 2011; Van der Cruyssen et al., 2014), and with multiple theorizations linking the processing of social concepts with affect (e.g., Lambon Ralph et al., 2016; Martin, 2016). Consistently with Simmons and Barsalou (2003), the present findings also support the distinction in the processing of positive and negative affective information, with the latter being implemented in the LIFGop. Finding the same pattern of results for positive words following the stimulation of an area involved in the processing of positive affective information would bring additional evidence in support of this distinction and in support of the hypothesis that social concepts rely on affective information. However, our results do not exclude the possibility that the LIFGop may not be the only area supporting the processing of social groups with a negative valence. In fact, the LIFGop is most likely a part of a wider network of cortical and subcortical regions supporting such processing, which the present stimulation may have affected indirectly. 
In conclusion, our data support the hypothesis of a predominant role of the LIFGop in affective processing, in particular of negative social groups. As such, our findings bring additional evidence to the theoretical propositions that link semantic representations to affective processes (Barsalou, 2008; Lambon Ralph et al., 2016; Martin, 2016; Simmons \& Barsalou, 2003) and suggest where in the brain this interplay is likely to take place. While a comparison with only the objects category poses a limit to the strength of our conclusions, we encourage future studies on the representation of social groups to replicate the present findings by taking into account comparisons with semantic categories such as animals. As previous research has argued objects and animals to be distinguished for the variety of their uses and of their visual properties, respectively, we argue that the factor playing a greater role in enabling us to discern among social groups is likely to be the affective valence we attribute to them. 


\section{REFERENCES}

Adolphs, R., Damasio, H., Tranel, D., Cooper, G., \& Damasio, a R. (2000). A role for somatosensory cortices in the visual recognition of emotion as revealed by three-dimensional lesion mapping. The Journal of Neuroscience, 20(7), 2683-2690. https://doi.org/123123123

Barsalou, L. W. (2008). Grounded Cognition. Annual Review of Psychology, 59(1), 617-645. https://doi.org/10.1146/annurev.psych.59.103006.093639

Capitani, E., Laiacona, M., Mahon, B., \& Caramazza, A. (2003). What are the facts of semantic category-specific deficits? A critical review of the clinical evidence. Cognitive Neuropsychology. https://doi.org/10.1080/02643290244000266

Caramazza, A., Anzellotti, S., Strnad, L., \& Lingnau, A. (2014). Embodied Cognition and Mirror Neurons: A Critical Assessment. Annual Review of Neuroscience. https://doi.org/10.1146/annurev-neuro-071013-013950

Carnaghi, A., Silveri, M. C., \& Rumiati, R. I. (2015). On the relationship between semantic knowledge and prejudice about social groups in patients with dementia. Cognitive and Behavioral Neurology, 28(2), 71-79. https://doi.org/10.1097/WNN.0000000000000059

Cattaneo, Z., Devlin, J. T., Salvini, F., Vecchi, T., \& Silvanto, J. (2010). The causal role of category-specific neuronal representations in the left ventral premotor cortex (PMv) in semantic processing. NeuroImage, 49(3), 2728-2734. https://doi.org/10.1016/j.neuroimage.2009.10.048

Cattaneo, Z., Rota, F., Vecchi, T., \& Silvanto, J. (2008). Using state-dependency of transcranial magnetic stimulation (TMS) to investigate letter selectivity in the left posterior parietal cortex: A comparison of TMS-priming and TMS-adaptation paradigms. European Journal of Neuroscience, 28(9), 1924-1929. https://doi.org/10.1111/j.1460-9568.2008.06466.x 
Chedid, G., Wilson, M. A., Provost, J. S., Joubert, S., Rouleau, I., \& Brambati, S. M. (2016). Differential involvement of the anterior temporal lobes in famous people semantics. Frontiers in Psychology, 7(AUG). https://doi.org/10.3389/fpsyg.2016.01333

Decety, J., \& Chaminade, T. (2003). Neural correlates of feeling sympathy. Neuropsychologia, 41(2), 127-138. https://doi.org/10.1016/S0028-3932(02)00143-4

Fuggetta, G., Rizzo, S., Pobric, G., Lavidor, M., \& Walsh, V. (2009). Functional Representation of Living and Nonliving Domains across the Cerebral Hemispheres: A Combined Event-related Potential/Transcranial Magnetic Stimulation Study. Journal of Cognitive Neuroscience, 21(2), 403-414. https://doi.org/10.1162/jocn.2008.21030

García-Pacios, J., Garcés, P., del Río, D., \& Maestú, F. (2017). Tracking the effect of emotional distraction in working memory brain networks: Evidence from an MEG study. Psychophysiology, 54(11), 1726-1740. https://doi.org/10.1111/psyp.12912

Haslam, C., \& Sabah, M. (2013). Preservation of person-specific knowledge in semantic memory disorder: A longitudinal investigation in two cases of dementia. Journal of Neuropsychology, 7(1), 132-138. https://doi.org/10.1111/j.1748-6653.2012.02030.x

Kay, J., \& Hanley, J. R. (2002). Preservation of memory for people in semantic memory disorder: Further category-specific semantic dissociation. Cognitive Neuropsychology, 19(2), 113-133. https://doi.org/10.1080/02643290143000114

Kensinger, E. A., \& Schacter, D. L. (2006). Processing emotional pictures and words: Effects of valence and arousal. Cognitive, Affective and Behavioral Neuroscience, 6(2), 110-126. https://doi.org/10.3758/CABN.6.2.110

Kissler, J., Assadollahi, R., \& Herbert, C. (2006). Chapter 8 Emotional and semantic networks in visual word processing: insights from ERP studies. Progress in Brain Research. 
https://doi.org/10.1016/S0079-6123(06)56008-X

Lambon Ralph, M. A., Jefferies, E., Patterson, K., \& Rogers, T. T. (2016). The neural and computational bases of semantic cognition. Nature Reviews Neuroscience. https://doi.org/10.1038/nrn.2016.150

Leclerc, C. M., \& Kensinger, E. A. (2011). Neural processing of emotional pictures and words: A comparison of young and older adults. Developmental Neuropsychology, 36(4), 519-538. https://doi.org/10.1080/87565641.2010.549864

Lyons, F., Kay, J., Hanley, J. R., \& Haslam, C. (2006). Selective preservation of memory for people in the context of semantic memory disorder: Patterns of association and dissociation. Neuropsychologia, 44(14), 2887-2898. https://doi.org/10.1016/j.neuropsychologia.2006.06.005

Mandera, P., Keuleers, E., \& Brysbaert, M. (2017). Explaining human performance in psycholinguistic tasks with models of semantic similarity based on prediction and counting: A review and empirical validation. Journal of Memory and Language, 92, 57-78. https://doi.org/10.1016/j.jml.2016.04.001

Martin, A. (2016). GRAPES - Grounding representations in action, perception, and emotion systems: How object properties and categories are represented in the human brain. Psychonomic Bulletin and Review, 23(4), 979-990. https://doi.org/10.3758/s13423-015-0842-3

Miceli, G., Capasso, R., Daniele, a, Esposito, T., Magarelli, M., \& Tomaiuolo, F. (2000). Selective deficit for people's names following left temporal damage: an impairment of domain-specific conceptual knowledge. Cognitive Neuropsychology, 17(6), 489-516. https://doi.org/10.1080/02643290050110629

Noonan, K. A., Jefferies, E., Visser, M., \& Lambon Ralph, M. A. (2013). Going beyond inferior 
prefrontal involvement in semantic control: evidence for the additional contribution of dorsal angular gyrus and posterior middle temporal cortex. Journal of Cognitive Neuroscience, 25(11), 1824-50. https://doi.org/10.1162/jocn_a_00442

Opie, G. M., Ridding, M. C., \& Semmler, J. G. (2015). Task-related changes in intracortical inhibition assessed with paired- and triple-pulse transcranial magnetic stimulation. Journal of Neurophysiology, 113(5), 1470-1479. https://doi.org/10.1152/jn.00651.2014

Patterson, K., Nestor, P. J., \& Rogers, T. T. (2007). Where do you know what you know? The representation of semantic knowledge in the human brain. Nature Reviews Neuroscience. https://doi.org/10.1038/nrn2277

Piretti, L., Carnaghi, A., Campanella, F., Ambron, E., Skrap, M., \& Rumiati, R. I. (2015). The neural network associated with lexical-semantic knowledge about social groups. Cortex, 70, 155-168. https://doi.org/10.1016/j.cortex.2015.06.024

Pridmore, S., Fernandes Filho, J. a, Nahas, Z., Liberatos, C., \& George, M. S. (1998). Motor threshold in transcranial magnetic stimulation: a comparison of a neurophysiological method and a visualization of movement method. The Journal of ECT.

Rogers, T. T., Lambon Ralph, M. A., Garrard, P., Bozeat, S., McClelland, J. L., Hodges, J. R., \& Patterson, K. (2004). Structure and Deterioration of Semantic Memory: A Neuropsychological and Computational Investigation. Psychological Review, 111(1), 205-235. https://doi.org/10.1037/0033-295X.111.1.205

Rossi, S., Hallett, M., Rossini, P. M., \& Pascual-Leone, A. Screening questionnaire before TMS: An update, 122 Clinical Neurophysiology 1686 (2011). https://doi.org/10.1016/j.clinph.2010.12.037

Rossi, S., Hallett, M., Rossini, P. M., Pascual-Leone, A., Avanzini, G., Bestmann, S., ... Ziemann, 
U. (2009). Safety, ethical considerations, and application guidelines for the use of transcranial magnetic stimulation in clinical practice and research. Clinical Neurophysiology. https://doi.org/10.1016/j.clinph.2009.08.016

Rumiati, R. I., Carnaghi, A., Improta, E., Diez, A. L., \& Silveri, M. C. (2014). Social groups have a representation of their own: Clues from neuropsychology. Cognitive Neuroscience, 5(2), 8596. https://doi.org/10.1080/17588928.2013.876981

Shamay-Tsoory, S. G., Aharon-Peretz, J., \& Perry, D. (2009). Two systems for empathy: A double dissociation between emotional and cognitive empathy in inferior frontal gyrus versus ventromedial prefrontal lesions. Brain, 132(3), 617-627. https://doi.org/10.1093/brain/awn279

Shirota, Y., Sommer, M., \& Paulus, W. (2016). Strength-Duration Relationship in Paired-pulse Transcranial Magnetic Stimulation (TMS) and Its Implications for Repetitive TMS. Brain Stimulation, 9(5), 755-761. https://doi.org/10.1016/j.brs.2016.04.019

Silvanto, J., Muggleton, N., \& Walsh, V. (2008). State-dependency in brain stimulation studies of perception and cognition. Trends in Cognitive Sciences, 12(12), 447-454. https://doi.org/10.1016/j.tics.2008.09.004

Simmons, W. K., \& Barsalou, L. W. (2003). The similarity-in-topography principle: Reconciling theories of conceptual deficits. Cognitive Neuropsychology. https://doi.org/10.1080/02643290342000032

Thompson-Schill, S. L. (2003). Neuroimaging studies of semantic memory: Inferring "how" from “where.” Neuropsychologia, 41(3), 280-292. https://doi.org/10.1016/S0028-3932(02)00161-6

Thompson, S. A., Graham, K. S., Williams, G., Patterson, K., Kapur, N., \& Hodges, J. R. (2004). Dissociating person-specific from general semantic knowledge: Roles of the left and right temporal lobes. Neuropsychologia, 42(3), 359-370. 
https://doi.org/10.1016/j.neuropsychologia.2003.08.004

Van der Cruyssen, L., Heleven, E., Ma, N., Vandekerckhove, M., \& Van Overwalle, F. (2014).

Distinct neural correlates of social categories and personality traits. NeuroImage, 104, 336346. https://doi.org/10.1016/j.neuroimage.2014.09.022 